\title{
A Computational Validation of Water Molecules Adsorption on an $\mathrm{NaCl}$ Surface
}

\author{
Xiao-Yan Liu, Jing-Wen Cao, Xiao-Ling Qin, Xu-Liang Zhu $\mathbb{1}$, Xu-Hao Yu, Xue-Chun Wang, Xiao-Qing Yuan, \\ Yu-He Liu, Yong Wang and Peng Zhang * (i)
}

check for

updates

Citation: Liu, X.-Y.; Cao, J.-W.; Qin, X.-L.; Zhu, X.-L.; Yu, X.-H.; Wang,

X.-C.; Yuan, X.-Q.; Liu, Y.-H.; Wang,

Y.; Zhang, P. A Computational

Validation of Water Molecules

Adsorption on an $\mathrm{NaCl}$ Surface.

Crystals 2021, 11, 610. https://

doi.org/10.3390/cryst11060610

Academic Editors: Tomoyuki

Hamada and Paolo Restuccia

Received: 17 April 2021

Accepted: 25 May 2021

Published: 28 May 2021

Publisher's Note: MDPI stays neutra with regard to jurisdictional claims in published maps and institutional affiliations.

Copyright: (c) 2021 by the authors. Licensee MDPI, Basel, Switzerland. This article is an open access article distributed under the terms and conditions of the Creative Commons Attribution (CC BY) license (https:/ / creativecommons.org/licenses/by/ $4.0 /)$.
School of Space Science and Physics, Shandong University, Weihai 264209, China; liuxiaoyan@mail.sdu.edu.cn (X.-Y.L.); caojw7689@mail.sdu.edu.cn (J.-W.C.); qinxl2582@mail.sdu.edu.cn (X.-L.Q.); zhuxuliang@outlook.com (X.-L.Z.); xhyu@mail.sdu.edu.cn (X.-H.Y.); xcwang@mail.sdu.edu.cn (X.-C.W.); yuanxiaoqing@mail.sdu.edu.cn (X.-Q.Y.); liuyuhe@mail.sdu.edu.cn (Y.-H.L.); wang.yong06@sdu.edu.cn (Y.W.) * Correspondence: zhangpeng@sdu.edu.cn

Abstract: It was reported that a scanning tunneling microscopy (STM) study observed the adsorption geometry of a water monomer and a tetramer on $\mathrm{NaCl}(100)$ film. Based on first-principles density functional theory (DFT), the adsorption behavior of water on the $\mathrm{NaCl}$ surface was simulated with CASTEP code. The results showed that the water monomer almost lay on the $\mathrm{NaCl}(001)$ surface with one $\mathrm{O}-\mathrm{H}$ bond tilted slightly downward. This was quite different from the STM observations. In fact, the experimental observation was influenced by the $\mathrm{Au}(111)$ substrate, which showed an upright form. A recent report on observations of two-dimensional ice structure on $\mathrm{Au}(111)$ substrate verified our simulations. However, the water tetramer formed a stable quadrate structure on the surface, which was consistent with observation. The intermolecular hydrogen bonds present more strength than surface adsorption. The simulations presented a clearer picture than experimental observations.

Keywords: first-principles; density functional theory; water; interface; $\mathrm{NaCl}$

\section{Introduction}

Although water is one of the most common molecules, study of the anomalous properties of water is still challenging. Many theoretical studies [1-4] and experimental observations $[5,6]$ have focused on the adsorption behavior of water molecules on the surface of transition and heavy metals. Water adsorption on an $\mathrm{NaCl}$ surface is also a typical system and is greatly significant to environmental science and biology systems.

Different computational methods have been used to investigate water behavior on an $\mathrm{NaCl}$ surface, such as classical molecular dynamics [7], ab initio simulation based on the cluster model [8], and density functional theory (DFT) with local density approximation (LDA) [9].

In laboratory experiments, Bruch et al. studied the adsorption of water on $\mathrm{NaCl}(001)$ using helium atom scattering and observed a diffraction pattern [10]. Verdaguer et al. used scanning polarized force microscopy to study the adsorption behavior of water molecules on cleaved $\mathrm{NaCl}(001)$ at different relative humidity [11]. Guo et al. used scanning tunneling microscopy (STM) to observe the water monomer and tetramer on an $\mathrm{NaCl}(001)$ surface with an Au substrate [12]. According to their report, a water monomer adsorbed on $\mathrm{NaCl}(001)$ film shows that the monomer was bonded on top of $\mathrm{Na}^{+}$and aligned with the $\mathrm{Na}^{+}-\mathrm{Cl}^{-}$direction. However, this may not be the true position of a water monomer adsorbed on a pure $\mathrm{NaCl}(001)$ surface. Meyer's simulation showed that water molecules took on almost horizontal adsorption configurations. For theoretical studies, determining whether LDA can correctly describe the adsorption of water molecules at an interface may be key to understanding the interaction between water and $\mathrm{NaCl}$ [13]. Yang et al. used the exchange correlation (XC) potential of a general gradient approximation (GGA) to study this issue and concluded that the water monomer dipole plane had a downward-tilted 
metastable configuration [14]. As the coverage rate of water molecules increases, the interaction between the water molecules is greatly enhanced, and the interaction between the water and $\mathrm{NaCl}$ surfaces decreases. Recently, Jiang et al. observed that 2D ice grown on the $\mathrm{Au}(111)$ surface corresponds to an interlocked bilayer ice structure consisting of two flat hexagonal water layers. They believed that bilayer hexagonal ice forms on hydrophobic substrate and that there is no hanging hydrogen bond on the surface of bilayer hexagonal ice [15]. Hereinafter, we simulated the monomer and tetramer geometry adsorption on an $\mathrm{NaCl}$ surface. The discrepancy between simulation and observation was also discussed.

\section{Materials and Methods}

The quantum mechanics DFT code CASTEP [16] was used to search the geometry configuration of water adsorption on $\mathrm{NaCl}$. The lattice constants and atomic coordinates of an $\mathrm{NaCl}$ unit cell are $\mathrm{a}=\mathrm{b}=\mathrm{c}=5.62 \AA$ and $\alpha=\beta=\gamma=90^{\circ}$ by default. The $\mathrm{NaCl}(001)$ plane was cleaved with a thickness of 6 layers, and a $3 \times 3$ supercell was then built with a vacuum of $12 \AA$. The water monomer was built separately and copied to the $\mathrm{NaCl}$ surface. The oxygen was set on the top of $\mathrm{Na}^{+}$. Considering the possibilities of upright to horizontal geometries, we designed seven different adsorption configurations. Considering that the electron density of this system is almost uniform, the XC functional of the LDA was used for geometric optimization with DFT + D correlations. Since the lattice constants should not be affected by water monomer, the supercell was not optimized. In addition, the inner-layer lattices were not constrained, meaning that the interactions between the water and surface went into three layers.

To mimic the STM experimental conditions, we further constructed an $\mathrm{Au}(111)$ substrate-based $\mathrm{NaCl}(001)$ bilayer surface according to Guo's experiments and repeated the water-adsorption calculation. First of all, the $\mathrm{Au}(111)$ plane and the $\mathrm{NaCl}(001)$ plane were cleaved separately. Since the lattice constants and atomic coordinates of $\mathrm{Au}(111)$ are $\mathrm{a}=\mathrm{b}=2.884 \AA$ and $\gamma=120^{\circ}$, the lattice constants of $\mathrm{NaCl}(001)$ and $\mathrm{Au}(111)$ are mismatched. Thus, the $\mathrm{Au}(111)$ plane was cleaved with a thickness of 2 layers, and a $9 \times 8$ supercell was built. The lattice constants and atomic coordinates of an Au unit cell are a $=25.954142 \AA$, $\mathrm{b}=23.070349 \AA$ and $\gamma=120^{\circ}$. Then, the lattice constants and atomic coordinates of $\mathrm{NaCl}$ were changed to $\mathrm{a}=25.133404 \AA, \mathrm{b}=22.480 \AA$ and $\gamma=116.565^{\circ}$ by justifying the surface vectors. A $2 \times 2$ supercell was built to match the lattice constants of $\mathrm{NaCl}(001)$ and $\mathrm{Au}(111)$. After that, the two-film structure was combined with a vacuum of $20 \AA$. Thus, we simulated the water monomer adsorption configuration on the $\mathrm{NaCl}-\mathrm{Au}$ surface.

Finally, a water tetramer was constructed and placed on the $\mathrm{NaCl}$ surface above four neighbor $\mathrm{Na}^{+}$ions. The geometry optimization was performed with the same parameters mentioned previously.

\section{Results and Discussion}

\subsection{Adsorption of Water Monomer on an $\mathrm{NaCl(001)}$ Surface}

For the modeling of the adsorption of water molecules on an $\mathrm{NaCl}(001)$ surface, we first placed a water molecule at about 2-3 $\AA$ above the $\mathrm{NaCl}(001)$ surface. The monomer configurations were designed with seven positions from upward to downward. Figure 1 shows the optimized geometries. Comparing the total system energy across these seven results, the structure with the lowest electrostatic potential was that one $\mathrm{O}-\mathrm{H}$ bond parallel to the $\mathrm{Na}-\mathrm{Cl}$ bond and the other hydrogen tilted slightly downward, as shown in Figure 1f. Figure 2 shows the energy differences curve and Table 1 presents values with the two $\mathrm{H}-\mathrm{Cl}$ lengths of these seven configurations. 


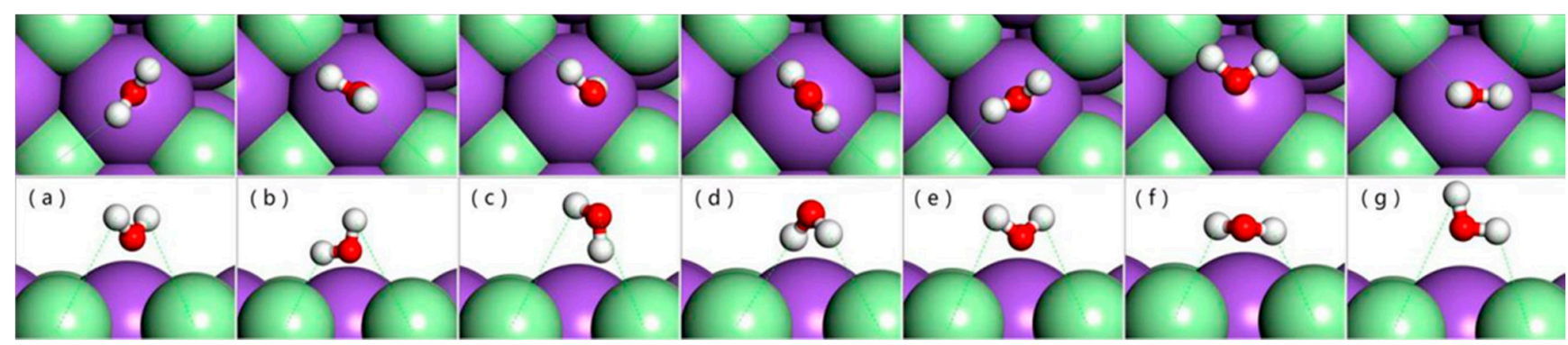

Figure 1. Seven conformations from (a-g) of a water monomer on an $\mathrm{NaCl}(001)$ surface. The upper picture is the top view and the lower picture is the side view for each optimized model. Conformation $\mathrm{f}$ has the lowest system energy. $\mathrm{O}, \mathrm{H}, \mathrm{Cl}^{-}$, and $\mathrm{Na}^{+}$are denoted by red, white, green, and purple spheres, respectively.

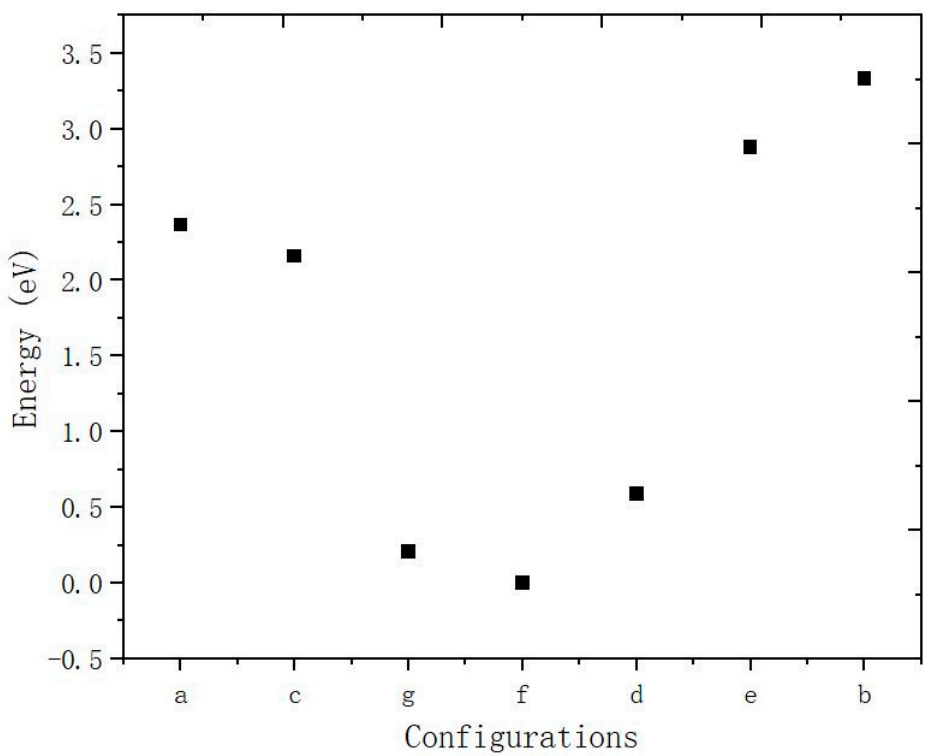

Figure 2. Energy differences across seven water monomer conformations. Structure $\mathrm{f}$ is the baseline and has the lowest system electrostatic potential.

Table 1. Energy difference (E) and optimized structural parameters of the seven models. The lowest system energy of conformation $\mathrm{f}$ was taken as the reference point. The two nearest $\mathrm{H}-\mathrm{Cl}$ distances are presented for comparison.

\begin{tabular}{cccc}
\hline Models & $\mathbf{E}(\mathbf{e V})$ & $\mathbf{H}^{\mathbf{1}} \mathbf{- C l}(\mathbf{\AA})$ & H-Cl (̊) \\
\hline a & 2.365 & 2.817 & 3.084 \\
b & 3.334 & 2.281 & 3.538 \\
c & 2.158 & 3.120 & 3.372 \\
d & 0.587 & 2.632 & 2.760 \\
e & 2.878 & 2.839 & 3.020 \\
f & 0 & 2.443 & 3.404 \\
g & 0.210 & 3.007 & 4.124 \\
\hline
\end{tabular}

Figure 3 shows the configuration of the f model in detail; the water molecule lies almost flat on the $\mathrm{NaCl}(001)$ surface, while the dipole plane is tilted slightly downward. In our simulation results, the thickness of $\mathrm{NaCl}$ film is $13.346 \AA$. The distance of $\mathrm{O}^{-}-\mathrm{Na}^{+}$ is $2.255 \AA$, and the distances of $\mathrm{H}^{+}-\mathrm{Cl}^{-}$are $2.220 \AA$ and $2.642 \AA$, respectively, showing a structure in which one $\mathrm{O}-\mathrm{H}$ bond is almost horizontal and the other is tilted slightly downward. In contrast, Yang's simulation showed that two O-H bonds were inclined downward at the same angle. The adsorptions of this system are derived from the intermolecular electrostatic interaction between polarized water molecules and $\mathrm{Na}^{+}-\mathrm{Cl}^{-}$ions. 
Due to the hybridization of the $\mathrm{SP}^{3}$ orbital of oxygen, the spatial distributions of the electron cloud present a tetrahedral structure along with two $\mathrm{O}-\mathrm{H}$ bonds. To match the interaction between $\mathrm{Na}^{+}$and the lone pair of oxygen electrons, the most stable configuration is slightly tilted. According to Guo's STM report, the water monomer was upright on the surface [12]. Since they only deposited a few layers of $\mathrm{NaCl}$ film, the upright geometry should be a result of repulsive forces from the $\mathrm{Au}$ substrate, and the actual interactions were $\mathrm{H}_{2} \mathrm{O} / \mathrm{NaCl} / \mathrm{Au}$. Thus, the real adsorption geometry configuration on the pure $\mathrm{NaCl}$ surface was not what they can see from STM experimental observations. We have discussed this issue with Jiang's team and confirmed this conclusion. Recently, their laboratory first observed twodimensional ice on $\mathrm{Au}(111)$ substrate [15]. The bilayer molecules bonded together to form a two-dimensional film. Each water molecule presents three inner-layer hydrogen bonds and one between-layers hydrogen bond. This phenomenon manifested the strong hydrophobic property of Au.

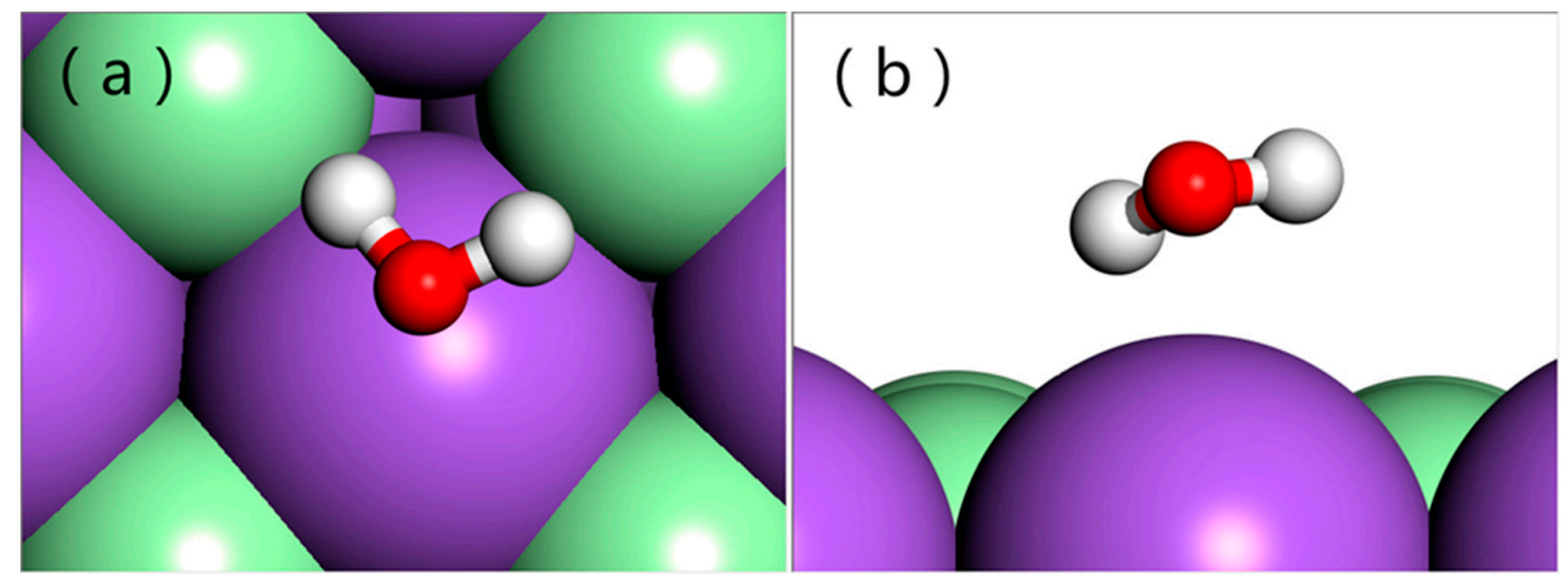

Figure 3. Refined structure of the water monomer on the $\mathrm{NaCl}(001)$ surface. Image (a) is the top view and (b) is the side view.

Subsequently, we simulated the adsorption configuration of water monomer on an $\mathrm{NaCl}(001)-\mathrm{Au}(111)$ surface. Figure 4 shows the configuration of the $\mathrm{H}_{2} \mathrm{O} / \mathrm{NaCl} / \mathrm{Au}$ model. This result was similar to Guo's experiment observation and confirm the conjecture above. The oxygen was on the top of $\mathrm{Na}^{+}$. One $\mathrm{O}-\mathrm{H}$ bond is almost vertical and the other is almost parallel to the $\mathrm{Na}^{+}-\mathrm{Cl}^{-}$bond. The distance of $\mathrm{H}_{2} \mathrm{O}-\mathrm{Na}^{+}$was changed from 2.255 to $2.469 \AA$. Comparison of Figures 3 and 4 shows the strong hydrophobic nature of Au.

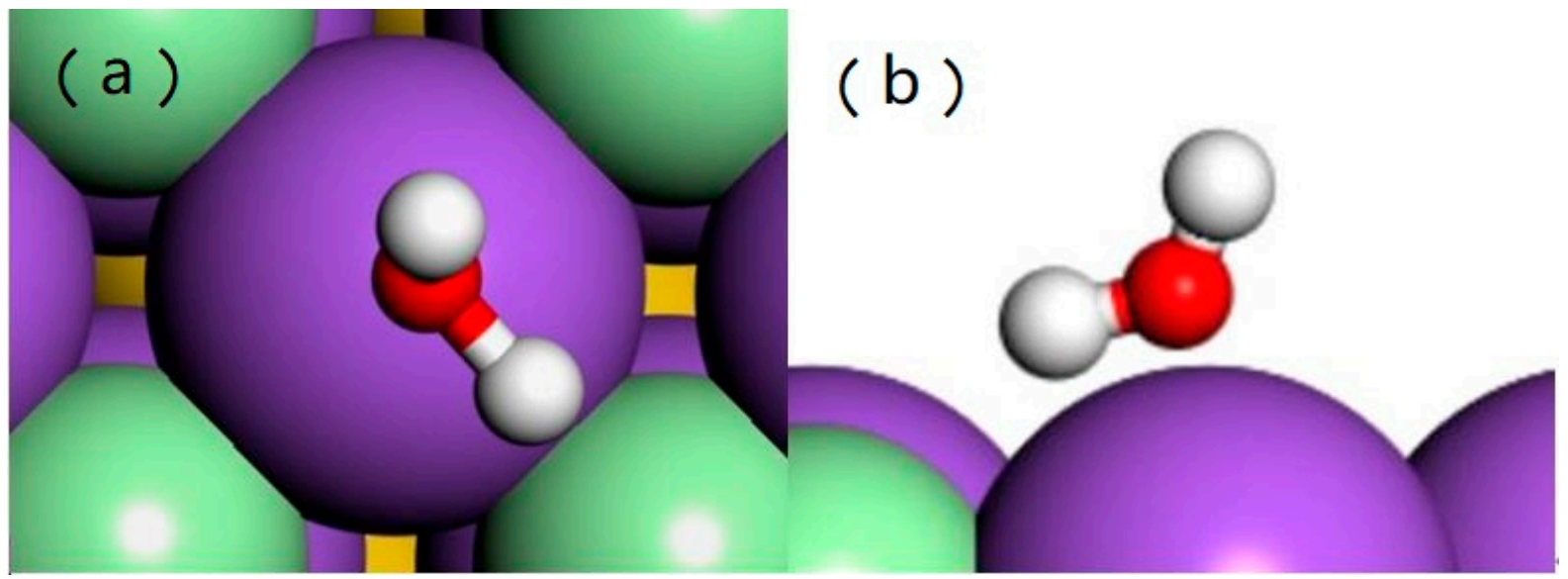

Figure 4. Refined structure of the water monomer on the $\mathrm{NaCl}(001)$ bilayer surface, based on $\mathrm{Au}(111)$ substrate. Image (a) is the top view and (b) is the side view. 


\subsection{Adsorption of Water Tetramer on an $\mathrm{NaCl(001)} \mathrm{Surface}$}

We then investigated the water cluster adsorption on the $\mathrm{NaCl}$ surface with the same calculation parameters. Four water molecules were placed arbitrarily above four $\mathrm{Na}^{+}$ions to form a square ring. As shown in Figure 5, each water molecule formed two hydrogen bonds as one proton donor and one proton receptor with two adjacent water molecules. Meanwhile, each oxygen atom closed to nearby $\mathrm{Na}^{+}$due to electrostatic interaction. Remarkably, each water molecule had a redundant hydrogen atom facing outward to achieve the tetrahedral configuration, due to the $\mathrm{SP}^{3}$ orbital hybridization of the oxygen atom.
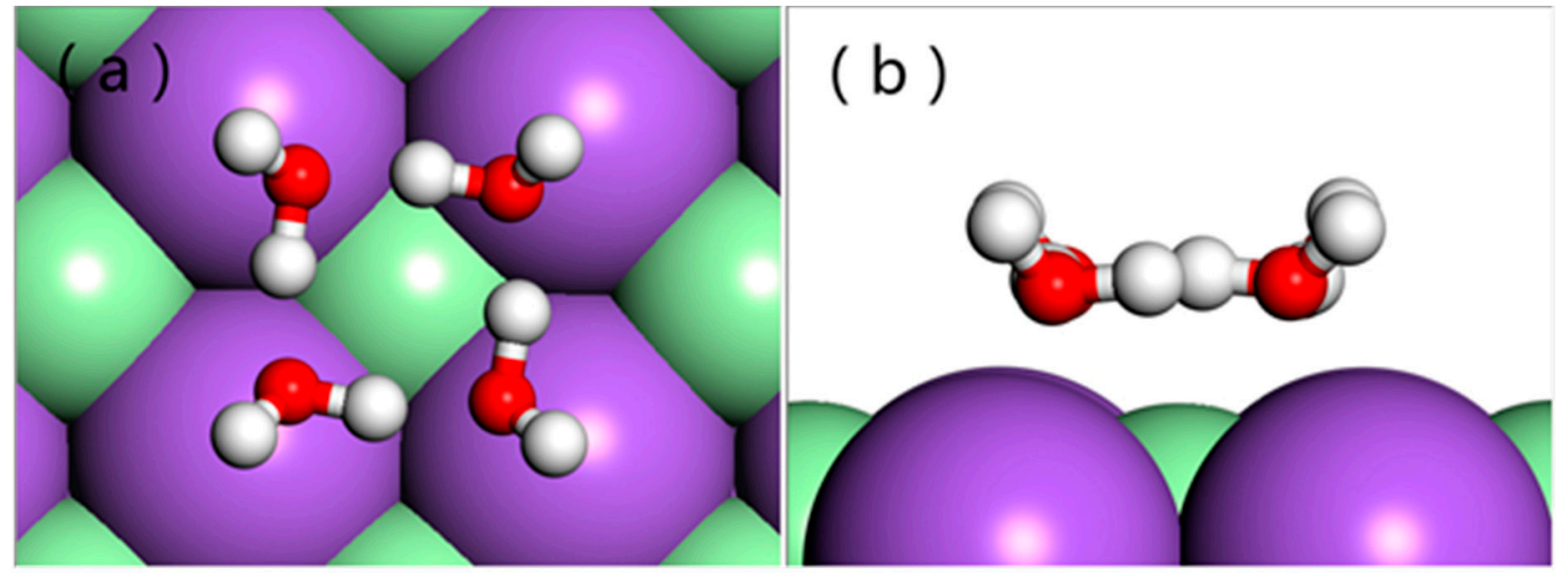

Figure 5. Optimized geometry structure of the water tetramer on the $\mathrm{NaCl}(001)$ surface. Image (a) is the top view and (b) is the side view.

Vitek et al. calculated the geometry structure of water tetramer [17]. Besides the four linked hydrogen bonds' inner four-molecule ring, the arrangement of the four dangling hydrogens is up-down-up-down with respect to the planar square cycle. Yang's simulations of water tetramer adsorption on the $\mathrm{NaCl}$ surface showed that the interactions with the interface were stronger, which manifested in two hydrogen atoms tilting downward to $\mathrm{Cl}^{-}$ ions. However, the STM observations of Guo et al. showed that the four water molecules took almost the same positions, showing good agreement with our simulations. Therefore, we confirmed that the hydrogen bonding occurred before the adsorption with $\mathrm{NaCl}$, as the hydrogen bonding was stronger than the other inter-molecular interactions.

\section{Conclusions}

Compared with STM observations, we confirmed that this computational work was more accurate than Yang's work. As they employed a GGA XC functional to calculate the interfacial interactions between water and $\mathrm{NaCl}$, it seemed that the LDA XC functional was more accurate than the GGA in this field. The DFT simulation configurations revealed an optimization scheme for the water wetting on the surface of non-metal substances. Moreover, owing to Jiang's advanced STM technology, we can verify the simulation results with directly experimental observations.

The calculation results present more precise information than the experiment. In the simulation of adsorption of water molecules on the $\mathrm{NaCl}(001)$ surface, one water monomer adsorbed on the $\mathrm{NaCl}(001)$ surface with one $\mathrm{O}-\mathrm{H}$ bond parallel to the $\mathrm{Na}^{+}-\mathrm{Cl}^{-}$bond, while the other hydrogen atom was tilted slightly downward. The water tetramer showed that the hydrogen bonding was stronger than the adsorption energy. Thus, we agree with Yang that the water tetramer is a possible building block for the adsorption of the first water cover layer on an $\mathrm{NaCl}$ surface. A new STM report by Jiang et al. observed two-dimensional ice on an $\mathrm{Au}(111)$ surface [15]. Also, we simulated the adsorption of water molecules on the $\mathrm{NaCl}(001)-\mathrm{Au}(111)$ surface. Compared with two results, it showed the hydrophobic nature between water and metal and the strong hydrogen bonding in- 
between water molecules. It also verified the monomer and tetramer geometries, both from the simulation and experiments.

Author Contributions: X.-Y.L. and J.-W.C. performed simulations; X.-L.Q., X.-L.Z., X.-H.Y. and X.-C.W. assisted with structural modeling and data processing; X.-Q.Y., Y.-H.L. participated in the discussion of results; Y.W. and P.Z. conducted the project and P.Z. edited the manuscript; all authors gave final approval for publication. All authors have read and agreed to the published version of the manuscript.

Funding: This research was funded by the National Natural Science Foundation of China, grant number 11075094.

Data Availability Statement: The data presented in this study are available on request from the corresponding author.

Acknowledgments: The numerical calculations were performed on the supercomputing system in the Supercomputing Center, Shandong University, Weihai.

Conflicts of Interest: The authors declare no conflict of interest.

\section{References}

1. Michaelides, A.; Ranea, V.A.; de Andres, P.L.; King, D.A. First-principles study of $\mathrm{H}_{2} \mathrm{O}$ diffusion on a metal surface: $\mathrm{H}_{2} \mathrm{O}$ on $\mathrm{Al}\{100\}$. Phys. Rev. Lett. 2003, 90, 216102. [CrossRef]

2. Meng, S.; Xu, L.F.; Wang, E.G.; Gao, S. Vibrational Recognition of Hydrogen-Bonded Water Networks on a Metal Surface. Phys. Rev. Lett. 2002, 89, 176104. [CrossRef] [PubMed]

3. Meng, S.; Wang, E.G.; Gao, S. A molecular picture of hydrophilic and hydrophobic interactions from ab initio density functional theory calculations. J. Chem. Phys. 2003, 119, 7617. [CrossRef]

4. Feibelman, P.J. Partial Dissociation of Water on Ru(0001). Science 2002, 295, 99. [CrossRef]

5. Morgenstern, K.; Nieminen, J. Intermolecular Bond Length of Ice on Ag(111). Phys. Rev. Lett. 2002, 88, 066102. [CrossRef]

6. Ogasawara, H.; Brena, B.; Nordlund, D.; Nyberg, M.; Pelmenschikov, A.; Pettersson, L.G.M.; Nilsson, A. Structure and Bonding of Water on Pt(111). Phys. Rev. Lett. 2002, 89, 276102. [CrossRef]

7. Wassermann, B.; Mirbt, S.; Reif, J.; Zink, J.C.; Matthias, E. Clustered water adsorption on the NaCl(100) surface. J. Chem. Phys. 1993, 98, 10049. [CrossRef]

8. Allouche, A. Water adsorption on $\mathrm{NaCl}(100)$ : A quantum ab-initio cluster calculation. Surf. Sci. 1998, 406, 279. [CrossRef]

9. Meyer, H.; Entel, P.; Hafner, J. Physisorption of water on salt surfaces. Surf. Sci. 2001, 488, 177. [CrossRef]

10. Bruch, L.W.; Glebov, A.; Toennies, J.P.; Weiss, H. A helium atom scattering study of water adsorption on the NaCl(100) single crystal surface. J. Chem. Phys. 2001, 103, 5109. [CrossRef]

11. Verdaguer, A.; Sacha, G.M.; Luna, M.; Ogletree, D.F.; Salmeron, M. Initial stages of water adsorption on NaCl(100) studied by scanning polarization force microscopy. J. Chem. Phys. 2005, 123, 124703. [CrossRef]

12. Guo, J.; Meng, X.; Chen, J.; Peng, J.; Sheng, J.; Li, X.-Z.; Xu, L.; Shi, J.-R.; Wang, E.; Jiang, Y. Real-space imaging of interfacial water with submolecular resolution. Nat. Mater. 2014, 13, 184-189. [CrossRef] [PubMed]

13. Giordano, L.; Goniakowski, J.; Suzanne, J. Partial Dissociation of Water Molecules in the (3×2) Water Monolayer Deposited on the $\mathrm{MgO}$ (100). Surf. Phys. Rev. Lett. 1998, 81, 1271. [CrossRef]

14. Yang, Y.; Meng, S.; Wang, E.G. Water adsorption on a $\mathrm{NaCl}$ (001) surface: A density functional theory study. Phys. Rev. B 2006, 74, 245409. [CrossRef]

15. Ma, R.; Cao, D.; Zhu, C.; Tian, Y.; Peng, J.; Guo, J.; Chen, J.; Li, X.Z.; Francisco, J.S.; Zeng, X.C. Atomic imaging of the edge structure and growth of a two-dimensional hexagonal ice. Nature 2020, 577, 60-63. [CrossRef] [PubMed]

16. Clark, S.J.; Segall, M.D.; Pickard, C.J.; Hasnip, P.J.; Probert, M.J.; Refson, K.; Payne, M.C. First Principles Methods Using CASTEP. Z. Kristallogr. 2005, 220, 567-570. [CrossRef]

17. Vitek, A.; Kalus, R.; Paidarova, I. Structural changes in the water tetramer. A combined Monte Carlo and DFT study. Phys. Chem. Chem. Phys. PCCP 2010, 12, 13657-13666. [CrossRef] [PubMed] 